\title{
A New Variable Star in Camelopardus.
}

A red star was detected 1887 Febr. 28 , and on March $I$ and 3 it was estimated $8^{\mathrm{m}} 2$ and $8^{\mathrm{m}}$.o, from the comparison stars: DM. $+68^{\circ} .40 \mathrm{r}$ Mag. 8.4 and DM. $+68^{\circ} 400$ Mag. 9.I while the red star was dentified as:

DM. $+68^{\circ} \cdot 39^{8} \quad \alpha=5^{\mathrm{h}} 25^{\mathrm{m} 22^{\mathrm{s}}} \quad \delta=+68^{\circ} 4^{2}: 5 \quad$ Mag. 9.3.

It was observed as $8 . \circ, 1887$ Nov. 14 . On the night of Febr. 16 I $89 \mathrm{I}$ it was found to be only $9 \mathrm{~m}$, and on Febr. I 99.2 . It was observed by Mr. Knott $189 \mathrm{r}$ Febr. 26 as 9.7 or $10 \mathrm{mo}$. The star is Es. B Brm. No. 146 . The star was believed to be IV type on one night, but on another, neither III nor IV, one very broad band and a suspicion of a bright line being noted.

Tow Law, Darlington, I89 I Febr. 28.

T. E. Espin.

Ephemeride für den Cometen 1890 II. (Forts. zu A. N. 3013.) $\mathbf{1 2}^{\mathbf{h}}$ M. Z. Berlin.

\begin{tabular}{|c|c|c|c|c|c|c|c|c|c|c|c|c|c|c|c|c|}
\hline \multirow{2}{*}{\multicolumn{2}{|c|}{$\frac{189 \text { I }}{\text { März } 2 \text { I }}$}} & \multicolumn{3}{|c|}{$\alpha$ app. } & \multicolumn{2}{|c|}{$\delta$ app. } & \multirow{2}{*}{$\frac{\log \gamma}{0.5857}$} & \multirow{2}{*}{$\underbrace{\log \Delta}_{0.4885}$} & \multirow{2}{*}{$\frac{H}{0.24}$} & \multirow{2}{*}{$\frac{189 I}{\text { April } 10}$} & \multicolumn{2}{|c|}{$\alpha$ app. } & $\delta$ app. & \multirow{2}{*}{$\frac{\log r}{0.605 \mathrm{I}}$} & \multirow{2}{*}{$\frac{\log \Delta}{0.5456}$} & \multirow{2}{*}{$\frac{H}{0.16}$} \\
\hline & & $10^{h}$ & $8^{m}$ & $3^{s}$ & $+35^{\circ}$ & $19: 5$ & & & & & $9^{h} 40^{n}$ & $3^{s}$ & $+3^{\circ} 5^{2: 6}$ & & & \\
\hline & 22 & & & 16 & 35 & 13.4 & & & & I I & 39 & 3 & $32 \quad 44 \cdot 4$ & & & \\
\hline & 23 & & 4 & 32 & 35 & 7.1 & & & & 12 & $3^{8}$ & 5 & 3236.1 & & & \\
\hline & 24 & & 2 & $5^{\circ}$ & 35 & 0.7 & & & & 13 & 37 & 9 & $\begin{array}{ll}32 & 27.8\end{array}$ & & & \\
\hline & 25 & ro & $\mathbf{I}$ & I I & 34 & 54.1 & $0.5^{8} 9^{6}$ & 0.4994 & 0.21 & I 4 & $3^{6}$ & I 4 & 32 I 9.5 & 0.6089 & 0.5573 & 0.15 \\
\hline & 26 & 9 & 59 & 35 & 34 & $47 \cdot 3$ & & & & I 5 & 35 & 2 I & $32 \times 1.1$ & & & \\
\hline & 27 & & $5^{8}$ & I & 34 & 40.4 & & & & 16 & 34 & $3 \mathrm{I}$ & $\begin{array}{ll}32 & 2.7\end{array}$ & & & \\
\hline & 28 & & $5^{6}$ & 29 & 34 & $33 \cdot 4$ & & & & I 7 & 33 & 43 & 3 I 54.2 & & & \\
\hline & 29 & & 54 & 59 & 34 & 26.2 & 0.5935 & 0.5106 & 0.19 & I 8 & 32 & 57 & 3145.7 & 0.6126 & 0.5690 & 0.14 \\
\hline & 30 & & 53 & $3^{2}$ & 34 & 18.9 & & & & 19 & 32 & I 3 & 3137.2 & & & \\
\hline & 31 & & $5^{2}$ & 7 & 34 & I 1.5 & & & & 20 & $3 I$ & 30 & $31 \quad 28.7$ & & & \\
\hline April & $\mathbf{I}$ & & $5^{\circ}$ & 44 & 34 & 4.0 & & & & $2 I$ & 30 & 49 & 3 I 20.2 & & & \\
\hline & 2 & & 49 & 24 & 33 & $5^{6.3}$ & 0.5974 & $0.522 \mathrm{I}$ & 0.18 & 22 & 30 & 10 & $\begin{array}{lll}31 & 1 & 1.8\end{array}$ & $0.6 \times 63$ & 0.5807 & 0.13 \\
\hline & 3 & & 48 & 6 & 33 & 48.6 & & & & 23 & 29 & 33 & 3 I 3.4 & & & \\
\hline & 4 & & 46 & 51 & 33 & 40.8 & & & & 24 & 28 & 57 & $30 \quad 55.0$ & & & \\
\hline & 5 & & 45 & $3^{8}$ & 33 & 32.9 & & & & 25 & 28 & 23 & $30 \quad 46.6$ & & & \\
\hline & 6 & & 44 & 26 & 33 & 25.0 & 0.6013 & $0.533^{8}$ & 0.17 & 26 & 27 & 51 & $30 \quad 3^{8.3}$ & 0.6200 & 0.5922 & 0.12 \\
\hline & 7 & & 43 & I 7 & 33 & 17.0 & & & & 27 & 27 & 30 & $30 \quad 30.0$ & & & \\
\hline & 8 & & 42 & 10 & 33 & 8.9 & & & & 28 & 26 & $5 I$ & $30 \quad 2 \times .8$ & & & \\
\hline & 9 & & $4 I$ & 5 & 33 & 0.8 & & & & 29 & 26 & 23 & $30 \quad 13.6$ & & & \\
\hline & 10 & 9 & 40 & 3 & +32 & $5^{2.6}$ & $0.605 \mathrm{I}$ & $0.545^{6}$ & 0.16 & 30 & 925 & $5^{6}$ & $+30 \quad 5.4$ & 0.6236 & 0.6035 & $0.1 \mathrm{I}$ \\
\hline
\end{tabular}

Als Einheit der Helligkeit ist jene des r 9. März 1890 gewählt worden.

Sternwarte Wien-Währing I 89 I Febr. 23.

Friedrich Bidschof.

Beobachtung des Planeten (305), entdeckt von Palisa 1891 Febr. 14.

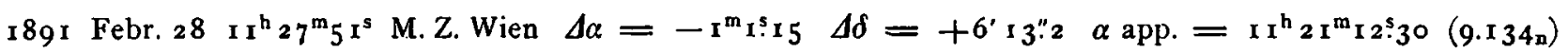
$\delta$ app. $=+8^{\circ} 5^{\prime} 23^{\prime \prime 2}$ (0.750) Red. ad l. app. $=+0.87-3^{\prime \prime} 4$

Vergleichstern (1891.o): $\alpha=$ ro $^{\mathrm{h}} 22^{\mathrm{m}}{ }^{\mathrm{r}} 2^{\mathrm{s}} 5^{8} \delta=+8^{\circ} 5^{2^{\prime}}$ 1 $3^{\prime \prime} 4$ A. N. Bd. I I X, S. 54 .

Wien 189 I März 2.

F. Palisa.

Beobachtungen des Planeten (306), entdeckt von Charlois 1891 Febr. 16.

\begin{tabular}{|c|c|c|c|c|c|c|c|c|}
\hline 1891 & M.Z. Ha mb. & $\Delta \alpha$ & $\Delta \delta$ & $a$ app. & $\log p \cdot \Delta$ & $\delta$ app. & $\log p \cdot 4$ & Red. ad 1. app. \\
\hline $\begin{array}{r}\text { Febr. } 26 \\
27\end{array}$ & $\begin{array}{lll}8^{h} & 19^{m} & 2^{s} \\
8 & 3^{2} & 35\end{array}$ & $\begin{array}{l}-2^{m} 18.60 \\
-259.68\end{array}$ & $\begin{array}{l}\text { - } 0^{\prime} 31.7 \\
+442.3\end{array}$ & 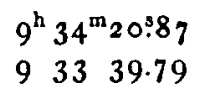 & $\begin{array}{l}9 \cdot 379 n \\
9 \cdot 338_{n}\end{array}$ & $\begin{array}{r}+7^{\circ} 53^{\prime 2.6} \\
+75826.6\end{array}$ & $\begin{array}{l}0.812 \\
0.809\end{array}$ & $\begin{array}{l}+0.82-2.5 \\
+0.82-2.5\end{array}$ \\
\hline
\end{tabular}

Die erste Beobachtung ist nach einem Telegramm bereits in Nr. 3022 mitgetheilt.

Sternwarte Hamburg 189 I Febr. 28.

W. Luther. 